\title{
Current-driven spin torque induced by the Rashba effect in a ferromagnetic metal layer
}

\author{
Ioan Mihai Miron ${ }^{1 \star}$, Gilles Gaudin ${ }^{2}$, Stéphane Auffret ${ }^{2}$, Bernard Rodmacq $^{2}$, Alain Schuhl ${ }^{2}$, \\ Stefania Pizzini ${ }^{3}$, Jan Vogel ${ }^{3}$ and Pietro Gambardella ${ }^{1,4}$
}

\begin{abstract}
Methods to manipulate the magnetization of ferromagnets by means of local electric fields ${ }^{1-3}$ or current-induced spin transfer torque ${ }^{4-6}$ allow the design of integrated spintronic devices with reduced dimensions and energy consumption compared with conventional magnetic field actuation ${ }^{7,8}$. An alternative way to induce a spin torque using an electric current has been proposed based on intrinsic spin-orbit magnetic fields ${ }^{9,10}$ and recently realized in a strained low-temperature ferromagnetic semiconductor ${ }^{11}$. Here we demonstrate that strong magnetic fields can be induced in ferromagnetic metal films lacking structure inversion symmetry through the Rashba effect. Owing to the combination of spin-orbit and exchange interactions, we show that an electric current flowing in the plane of a Co layer with asymmetric $\mathrm{Pt}$ and $\mathrm{AlO}_{x}$ interfaces produces an effective transverse magnetic field of $1 \mathrm{~T}$ per $10^{8} \mathrm{~A} \mathrm{~cm}^{-2}$. Besides its fundamental significance, the high efficiency of this process makes it a realistic candidate for room-temperature spintronic applications.
\end{abstract}

Controlling the magnetization of nanodevices using local electric currents instead of externally applied magnetic fields opens a wide spectrum of opportunities to integrate magnetic functionalities into electronic circuits. In general, current-induced magnetization reversal requires the generation of a conduction electron spin density non-collinear with the local magnetic moments of a ferromagnet. This is achieved in multilayer structures such as spin valves and tunnel junctions by injecting spin-polarized electrons from a pinned ferromagnet into a 'free' ferromagnetic layer through a non-magnetic spacer, inducing the so-called spintransfer-torque (STT) effect ${ }^{6,12,13}$.

An alternative way to produce non-equilibrium polarization of the conduction electron spins is based on spin-orbit coupling in structures lacking inversion symmetry. Electrons moving in an asymmetric crystal-field potential experience a net electric field (E), which, by transforming into a magnetic field in the electron's rest frame, effectively couples the spin to the electron's orbital motion $^{14,15}$. This effect is well known for semiconductors, where the occurrence of net spin polarization has been predicted ${ }^{16,17}$ and observed in electric currents flowing through non-magnetic quantum wells ${ }^{18}$ as well as strained bulk compounds ${ }^{19}$.

Recent theoretical work suggests that current-induced spinorbit effects detected in non-magnetic semiconductors ${ }^{20}$ could be used in combination with ${ }^{21}$ or be intrinsic to ferromagnets ${ }^{9,10,22}$, providing a radically new mechanism for the manipulation of the magnetization in ferromagnetic systems. Of interest here is the effective magnetic field produced by the spin-orbit interaction on the conduction electrons of a two-dimensional system characterized by structural inversion asymmetry (SIA). This field, named after Rashba $^{15}$, is given by $H_{\mathbf{R}}=\alpha_{\mathrm{R}}(\hat{\mathbf{z}} \times\langle\mathbf{k}\rangle)$, where $\hat{\mathbf{z}}$ is a unit vector parallel to $\mathbf{E},\langle\mathbf{k}\rangle$ is the average electron wavevector and $\alpha_{\mathrm{R}}$ is a material parameter that depends on the strength of the spin-orbit coupling. For zero current, $H_{\mathrm{R}}$ cancels out as $\mathbf{k}$ and $-\mathbf{k}$ states are equally populated and $\langle\mathbf{k}\rangle=0$. In the presence of a charge current, however, the electron distribution in $\mathbf{k}$-space becomes asymmetric, producing a net effective field and inducing a nonequilibrium spin density perpendicular to the current direction. In a ferromagnet, the action of $H_{\mathrm{R}}$ combines with the $s-d$ exchange interaction that couples the conduction electron spin to the local magnetization $(M)$. As a result of this interaction a current passing through a uniformly magnetized ferromagnet layer has been predicted to exert an exchange-mediated effective field $H_{\text {sd }}$ on $M$ (Fig. 1a), thereby producing a torque and eventually provoking the reversal of $M$ (refs 9, 10). A similar mechanism, albeit based on strain-induced Dresselhaus spin-orbit coupling ${ }^{14,23}$ rather than the Rashba effect, has very recently been demonstrated in a p-type ferromagnetic semiconductor, $\mathrm{Ga}_{0.94} \mathrm{Mn}_{0.06} \mathrm{As}$, where the injection of $10^{6} \mathrm{~A} \mathrm{~cm}^{-2}$ produced an effective field of about $0.5 \mathrm{mT}$ at $T=40 \mathrm{~K}$ (ref. 11). It should be noticed that a spin-orbitinduced torque is fundamentally different from STT, although its ultimate effects might be similar. Contrary to STT, there is no transfer of spin angular momentum from one ferromagnetic layer to another. Rather, orbital angular momentum is transferred to the electron spins through the spin-orbit interaction intrinsic to the band structure of the ferromagnet. Thus, the application of a spin torque does not rely on two non-collinear, independently controlled ferromagnet electrodes and can be realized in a single uniformly magnetized ferromagnetic layer. Moreover, the direction of the spin-orbit torque may be changed by reversing the current, thereby enabling reversible control of the magnetization.

Although studies of the Rashba effect have traditionally focused on semiconducting materials, several groups have now reported evidence of Rashba-induced splitting of the surface states of both non-magnetic ${ }^{24,25}$ and magnetic metals ${ }^{26}$ using angle-resolved photoemission. All of the elements required by theory ${ }^{9,10}$ have therefore been separately observed, but spin-torque effects in metallic ferromagnetic systems have not yet been realized. Here, we report on the observation of a current-induced spin-orbit torque in a uniformly magnetized ferromagnetic metal layer with enhanced SIA. We demonstrate that this process arises from the Rashba effect at the interface of the ferromagnetic layer and determine the magnitude of the effective field $H_{s d}$ as a function of current density.

Experiments were carried out on a 0.6-nm-thick Co layer sandwiched between $3 \mathrm{~nm}$ Pt and $1.6 \mathrm{~nm} \mathrm{Al} \mathrm{films} \mathrm{deposited} \mathrm{on}$

${ }^{1}$ Centre d'Investigació en Nanociència i Nanotecnologia (ICN-CSIC), UAB Campus, E-08193 Barcelona, Spain, ${ }^{2}$ SPINTEC, UMR-8191, 
a

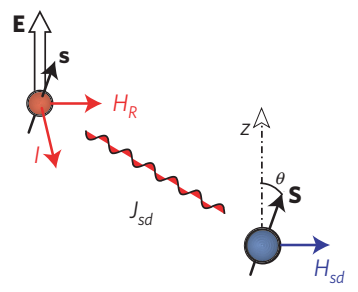

c

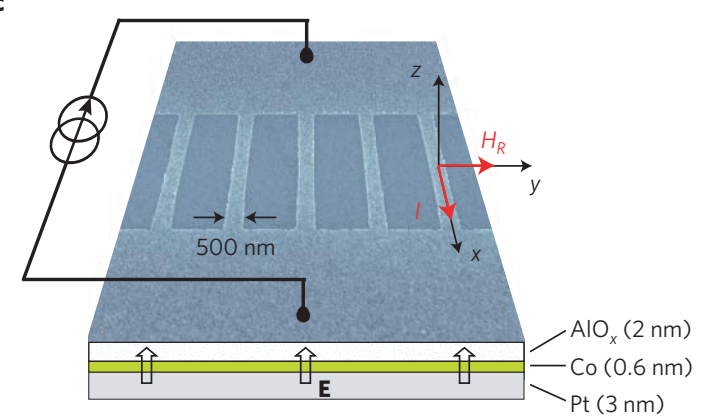

b

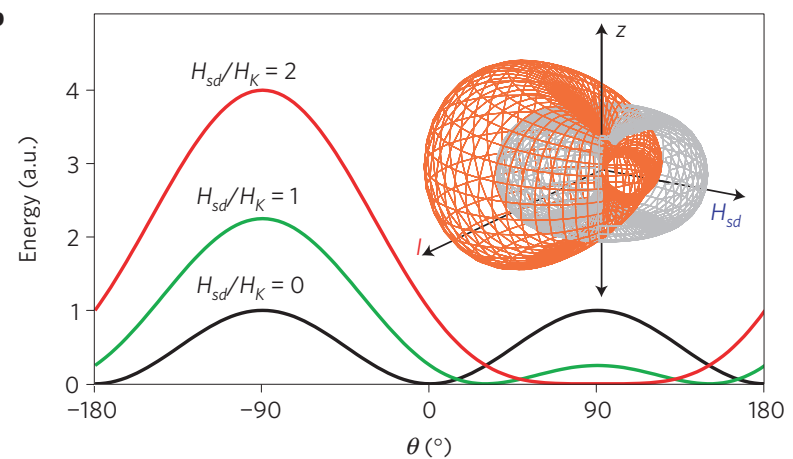

d

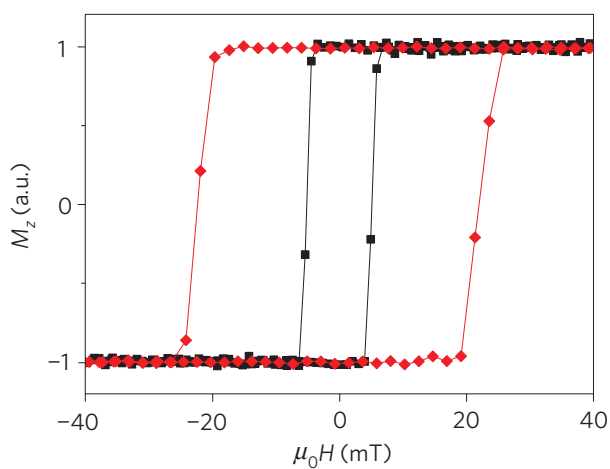

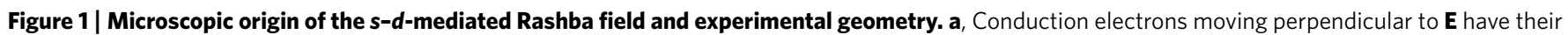
spin (s) tilted by the Rashba magnetic field $\left(H_{R}\right)$, exerting a torque on the localized moments $(\mathbf{S})$ through the exchange coupling $\left(J_{s}\right)$ and $s-d$-mediated Rashba field $\left(H_{s d}\right)$. b. Similarly to an external magnetic field, this interaction reduces the energy barrier for magnetization reversal in the direction

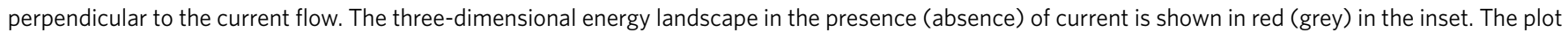
shows the magnetization energy as a function of polar angle $\theta$ calculated for a magnetic layer with the uniaxial anisotropy easy axis parallel to $\hat{\mathbf{z}}$ and current flow along $\hat{\mathbf{x}}$. Both the equilibrium magnetization direction and barrier height depend on the ratio between the effective anisotropy field $\left(H_{K}\right)$ and $H_{s d}$. c, Scanning electron micrograph detail of the patterned $\mathrm{Pt} / \mathrm{Co} / \mathrm{AlO}_{x}$ wire array and schematic vertical cross-section of the layer. Arrows indicates the

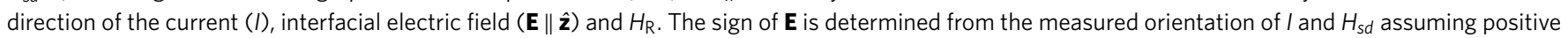

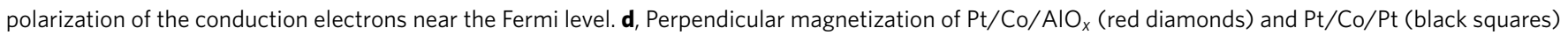
measured at room temperature.

a thermally oxidized $\mathrm{Si}$ wafer. The top Al layer was further exposed to an oxygen radiofrequency plasma resulting in a fully oxidized $\mathrm{Co} / \mathrm{AlO}_{x}$ interface, as described in ref. 27. SIA results from the presence of $\mathrm{AlO}_{x}$ and $\mathrm{Pt}$ on either side of the Co layer. Photoemission and magnetic dichroism studies have shown that spin-orbit effects at metal surfaces increase with the presence of heavy atoms ${ }^{28,29}$ and surface oxidation ${ }^{24}$. Here, both were used to create a strong out-of-plane electron potential gradient in $\mathrm{Pt} / \mathrm{Co} / \mathrm{AlO}_{x}$, as also suggested by the enhanced non-adiabatic spin-torque component found in domain wall constrictions $\mathrm{s}^{30}$. The Co magnetization has a saturation value close to the bulk, $M=1,090 \mathrm{kA} \mathrm{m}^{-1}$, and is very stable after oxidation. Extraordinary Hall effect measurements evidence strong perpendicular anisotropy and show that $\mathrm{Pt} / \mathrm{Co} / \mathrm{AlO}_{x}$ exhibits a uniform monodomain state at remanence (Fig. 1d). The out-of-plane uniaxial anisotropy field is $\mu_{0} H_{K}=0.92 \mathrm{~T}$, determined as described in the Supplementary Information (Supplementary Fig. S1). All measurements were carried out in ambient conditions. To observe the effects of an electric current on $M$, the $\mathrm{Pt} / \mathrm{Co} / \mathrm{AlO}_{x}$ film was patterned into an array of 19 wires, each $0.5 \mu \mathrm{m}$ wide and $5 \mu \mathrm{m}$ long, and contacted by two current pads (Fig. 1c). In this geometry, the application of a current is expected to produce an in-plane field $H_{\text {sd }}$ perpendicular to the wires, analogous to an external field applied along $\hat{y}$. Depending on the $H_{s d} / H_{K}$ ratio, the energy barrier for magnetization reversal will thus change from the initial symmetric shape typical of uniaxial anisotropy to a strongly asymmetric profile, lowering the barrier in one direction and raising it in the opposite one as the equilibrium magnetization direction gradually shifts from perpendicular to in-plane (Fig. 1b).

The magnetization of the wires was monitored using wide-field polar Kerr microscopy. Starting from a fully saturated out-of-plane $M$, single current pulses of increasing amplitude and constant $100 \mathrm{~ns}$ duration were injected into the wires until the nucleation of reversed domains was observed (Supplementary Fig. S2). This occurred as the wires evolved from the metastable monodomain state towards the macroscopically demagnetized ground state constituted by an equal mixture of up and down domains. To evidence the presence of $H_{s d}$, we introduced an external in-plane field $H_{\text {ext }}$ acting as a bias parallel or antiparallel to $\hat{y}$. Figure 2 shows a series of differential Kerr images recorded after injecting a positive current pulse of $7.8 \times 10^{7} \mathrm{~A} \mathrm{~cm}^{-2}$ in a $\mathrm{Pt} / \mathrm{Co} / \mathrm{AlO}_{x}$ wire array initially saturated along $-\hat{\mathbf{z}}$. At $\mu_{0} H_{\text {ext }}=0$, local changes of Kerr contrast indicate that reverse domain nucleation has occurred in about half of the wires. Surprisingly, all wires have nucleated domains for $\mu_{0} H_{\text {ext }}=+47.5 \mathrm{mT}$ but no evidence of magnetization reversal was observed when $\mu_{0} H_{\text {ext }}=-47.5 \mathrm{mT}$. This asymmetry cannot be reconciled either with the action of the Oersted field, which is parallel to $-\hat{\mathbf{y}}$ and smaller than $1 \mathrm{mT}$, or with simple thermal activation owing to Joule heating. To prove whether the observed behaviour is associated with the direction of electron motion, the same measurements were repeated for negative currents. The comparison of Fig. $2 \mathrm{a}$ and $\mathrm{b}$ shows that, when $\mu_{0} H_{\mathrm{ext}}=0$, positive and negative current directions yield a similar amount of nucleation events. However, for a fixed $H_{\text {ext }}$, the 

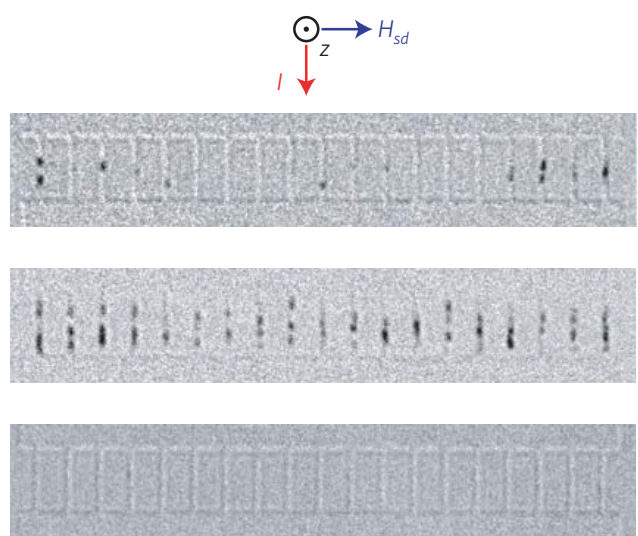

b
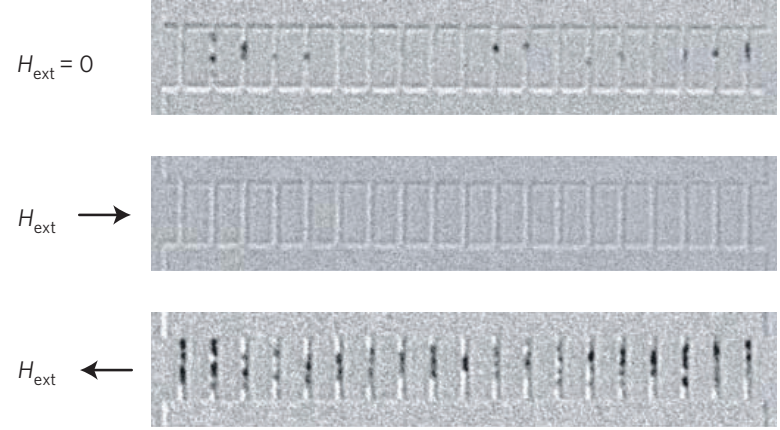

Figure 2 | Differential Kerr microscopy images recorded after current pulse injection. a,b, Positive (a) and negative (b) current values with external field $\mu_{0} H_{\text {ext }}=0, \pm 47.5 \mathrm{mT}$ applied parallel to $\hat{\mathbf{y}}$. The pulse amplitude is set to $7.8 \times 10^{7} \mathrm{~A} \mathrm{~cm}^{-2}$ in all cases. Note that the topographic contrast in each image varies depending on the drift between pre-pulse and post-pulse images.
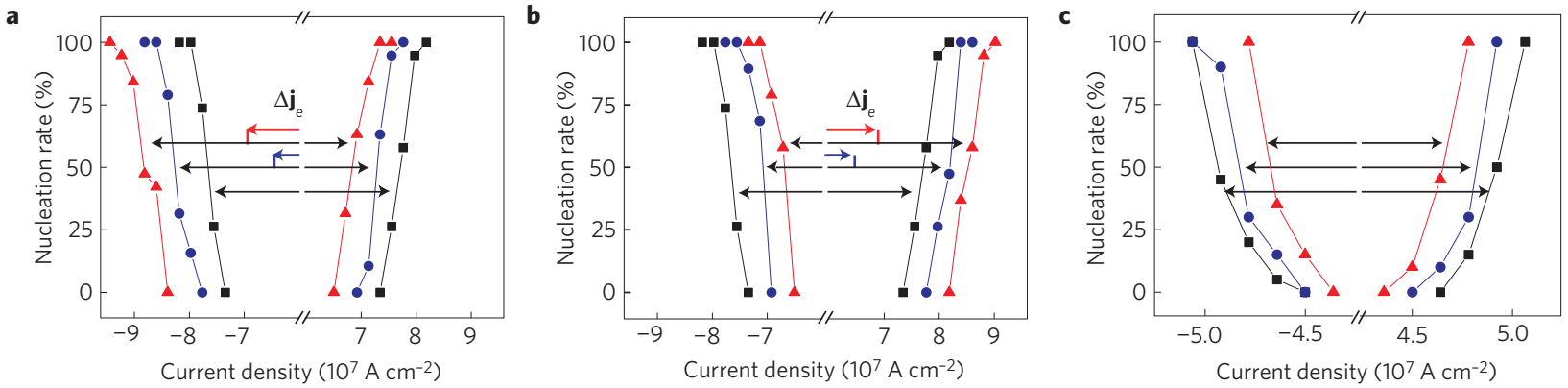

Figure 3 | Percentage of wires that present reversed magnetic domains after the injection of a current pulse as a function of current density and external field. a,b, $\mathrm{Pt} / \mathrm{Co} / \mathrm{AlO}_{x}$ wire array for $\mathrm{H}_{\mathrm{ext}} \| \hat{\mathbf{y}}(\mathbf{a})$ and $H_{\mathrm{ext}} \|-\hat{\mathbf{y}}(\mathbf{b})$. c, $\mathrm{Pt} / \mathrm{Co} / \mathrm{Pt}$ wire array, $\mathbf{H}_{\mathrm{ext}} \|-\hat{\mathbf{y}}$. The nucleation rate curves shift by an amount $\Delta \mathbf{j}_{\mathrm{e}}$ proportional to $H_{\text {ext }}$, as indicated by red and blue arrows, reflecting the direction and magnitude of $H_{s d}$. Values of $\mu_{0} H_{\text {ext }}$ are 0 mT (black squares), $\pm 47.5 \mathrm{mT}$ (blue dots), $\pm 95 \mathrm{mT}$ (red triangles).

effect of the current sign becomes obvious leading to either strong amplification or suppression of domain nucleation depending on the orientation of the current density vector $\mathbf{j}_{\mathrm{e}}$. Experiments repeated with $M$ initially saturated along $\hat{\mathbf{z}}$ showed that this behaviour is independent of $M$ being up or down (Supplementary Fig. S3). These measurements, carried out at constant current density, exclude thermal effects as the origin of the observed domain nucleation rate asymmetry. Furthermore, artefacts owing to a small unintentional misalignment of $H_{\text {ext }}$ outside the $x y$ plane may also be ruled out, as these would be independent of the sign of $\mathbf{j}_{\mathrm{e}}$.

These results qualitatively prove the presence of a currentinduced torque acting on $M$ with the symmetry properties predicted by theory ${ }^{9,10}$. To quantitatively determine the dependence of $H_{s d}$ on $\mathbf{j}_{\mathrm{e}}$, we make systematic use of $H_{\text {ext }}$ as a known reference field, plotting in Fig. 3 the percentage of wires for which at least one nucleation event is observed for a given combination of $\left(H_{\mathrm{ext}}, \mathbf{j}_{\mathrm{e}}\right)$. In agreement with the above behaviour, we find that the nucleation rate obtained in the absence of external field is symmetric with respect to $\mathbf{j}_{\mathrm{e}}$, whereas for $H_{\text {ext }} \neq 0$ curves corresponding to opposite current polarity shift proportionally to $H_{\text {ext }}$. The sign of the shift depends on the orientation of $H_{\text {ext }}$ as more or less current is required to attain a given nucleation rate when $H_{\text {ext }}$ opposes or favours $H_{s d}$. Note that other criteria for quantifying the nucleation of reversed domains have been tested, such as the total area of reversed Kerr contrast, and yield equivalent results to Fig. 3.

Figure 4 shows that the magnitude of the current shift is a linear function of $H_{\text {ext }}$. As $H_{s d}$ acts analogously to an external field, the inverse slope of the linear fit in Fig. 4 provides a direct estimate of the $H_{s d} / \mathbf{j}_{\mathrm{e}}$ ratio, yielding $(1.0 \pm 0.1) \times 10^{-8} \mathrm{~T} \mathrm{~cm}^{2} \mathrm{~A}^{-1}$. This value can be compared to the magnitude of the field predicted in ref. 9 ,

$$
\mu_{0} H_{s d} \approx \frac{\alpha_{\mathrm{R}}}{\mu_{\mathrm{B}} M} P\left(\hat{\mathbf{z}} \times \mathbf{j}_{\mathbf{e}}\right)
$$

where $\mu_{\mathrm{B}}$ is the Bohr magneton and $P$ is a parameter that depends on the $s-d$ coupling strength, which can be approximated by the degree of polarization of the conduction electrons. After substituting $P \approx 0.5$ and $M=1.09 \times 10^{6} \mathrm{~A} \mathrm{~m}^{-1}$ for the Co layer in $\mathrm{Pt} / \mathrm{Co} / \mathrm{AlO}_{x}$, our data agree with equation (1) provided that we assume $\alpha_{\mathrm{R}}=10^{-10} \mathrm{eV} \mathrm{m}$, which is a realistic estimate considering that $\alpha_{\mathrm{R}}$ ranges from $4 \times 10^{-11}$ to $3 \times 10^{-10} \mathrm{eV} \mathrm{m}$ at the interface of heavy-metal systems ${ }^{25,29}$ and that oxidation further enhances $\alpha_{\mathrm{R}}$ (ref. 26).

To further check whether the observed current-field relationship is associated with the SIA of the layer structure, as expected for the Rashba effect, we have carried out a control experiment on a symmetric $\mathrm{Pt} / \mathrm{Co} / \mathrm{Pt}$ structure, replacing $\mathrm{AlO}_{x}$ by a 3 -nm-thick $\mathrm{Pt}$ film. Similarly to $\mathrm{Pt} / \mathrm{Co} / \mathrm{AlO}_{x}, \mathrm{Pt} / \mathrm{Co} / \mathrm{Pt}$ presents strong outof-plane anisotropy and uniaxial anisotropy field $\mu_{0} H_{K}=0.57 \mathrm{~T}$, as reported in Fig. 1d and Supplementary Fig. S1. In Pt/Co/Pt, however, we detected no significant shift of the nucleation rate depending on the sign of $\mathbf{j}_{\mathrm{e}}$ (Fig. 3c) or initial saturation direction (Fig. 4). We thus prove that SIA is required to produce an in-plane effective field and confirm the Rashba field scenario.

The action of $H_{s d}$ enters into the dynamic equation of motion of the magnetization on equal footing with an external field and is independent of the magnetic configuration of the layer. Thus, 


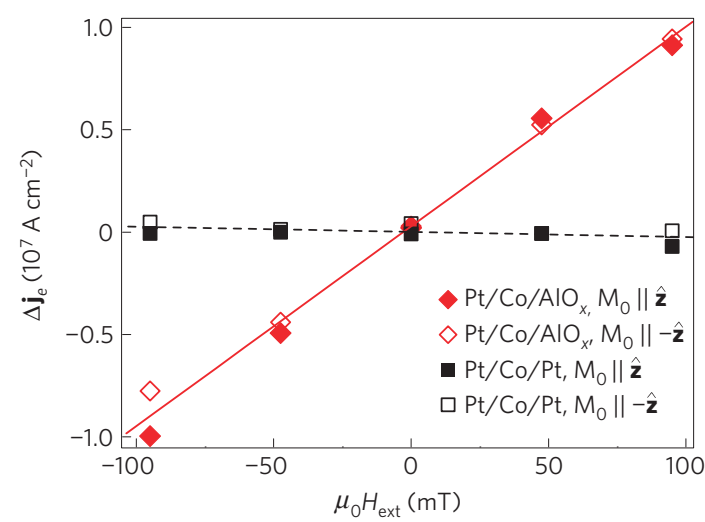

Figure 4 | Current density to magnetic field relationship. The red diamonds correspond to $\mathrm{Pt} / \mathrm{Co} / \mathrm{AlO}_{x}$ and the black squares to $\mathrm{Pt} / \mathrm{Co} / \mathrm{Pt}$. The filled and open symbols refer to samples initially magnetized up $(\| \hat{\mathbf{z}})$ and down $(\|-\hat{\mathbf{z}})$, respectively. The lines are linear fits to the data.

as discussed in the Supplementary Information, $H_{s d}$ produces a torque that is qualitatively different from other known types of current-induced torque, including the enhanced form of the non-adiabatic spin torque attributed to SIA in $\mathrm{Pt} / \mathrm{Co} / \mathrm{AlO}_{x}$ domain wall constriction $\mathrm{s}^{30}$, and provides new and flexible means to control the magnetic behaviour of metal systems.

Although the origin of the spin-orbit-induced magnetic fields is different, the comparison of $\mathrm{Pt} / \mathrm{Co} / \mathrm{AlO}_{x}$ and $\mathrm{Ga}_{0.94} \mathrm{Mn}_{0.06} \mathrm{As}$ reveals that spin manipulation through intrinsic relativistic effects is possible in metals and semiconductors alike. Remarkably, for the same current density, $H_{s d}$ is a factor of 20 larger in $\mathrm{Pt} / \mathrm{Co} / \mathrm{AlO}_{x}$ relative to $\mathrm{Ga}_{0.94} \mathrm{Mn}_{0.06} \mathrm{As}$, which we attribute to the disparity of Rashba and linear Dresselhaus coefficients ${ }^{11,23}$, as well as differences in the charge carrier density, $s-d$ exchange and saturation magnetization of the two materials ${ }^{9,10}$. Moreover, the $\mathbf{k}$ dependence of the Rashba and Dresselhaus terms is such that $H_{s d}$ is always orthogonal to $\mathbf{j}_{\mathrm{e}}$ in our case, whereas both transverse and longitudinal components are present in $\mathrm{Ga}_{0.94} \mathrm{Mn}_{0.06}$ As depending on the angle of $j_{\text {e }}$ relative to the crystallographic axes ${ }^{11}$. It shall be noticed that dilute magnetic semiconductors still face severe limitations for practical uses owing to their low Curie temperature, metastable crystal structure and high production costs. Ferromagnetic metals, on the other hand, offer reliable room-temperature operation, cheap fabrication and may be readily integrated in existing technological platforms.

For current densities $\mathbf{j}_{\mathrm{e}} \sim 10^{8} \mathrm{Acm}^{-2}$ comparable to those required by STT device operation ${ }^{4,6-8}$, our results show that extraordinarily high effective fields of the order of $1 \mathrm{~T}$ can be attained. If $H_{s d}$ is set orthogonal to $M$ by sending a current perpendicular to the easy magnetization axis, as in our case, the energy barrier separating the two stable states is reduced. On the other hand, if $H_{K}$ and $H_{s d}$ are engineered to be collinear, a favourable situation for achieving deterministic switching may be realized $^{11}$. A major advantage of the Rashba torque mechanisms is that the thermal stability of the magnetization, determined by $H_{K}$, and spin-torque efficiency, determined by $\alpha_{\mathrm{R}}$, scale together as both depend on the strength of the spin-orbit interaction. Equation (1) shows that, by fabricating alloys with increased $\alpha_{\mathrm{R}}$ (ref. 29) and careful balancing of magnetocrystalline and shape anisotropy, the critical current density required to induce magnetization reversal may be decreased to well below $10^{7} \mathrm{~A} \mathrm{~cm}^{-2}$.

We reported the experimental observation of spin-orbit torque induced by an electric current flowing through a uniformly magnetized ferromagnetic metal layer owing to the Rashba effect. This process originates from SIA and is mediated by the spin-orbit interaction in combination with $s-d$ exchange, allowing for the transfer of orbital angular momentum from the crystal lattice to the local spin magnetization. The measured value of the effective Rashba field acting on $M$ is very large, making for one of the most efficient spin-torque effects evidenced so far. As this is a current-driven mechanism and the layer structure is very similar to the lower electrode of a magnetic tunnel junction, devices combining Rashba and STT effects can also be designed. The simple layer structure, robust ferromagnetic properties of metal films and the possibility of modulating the Rashba field by an external gate voltage ${ }^{9}$ open a promising new avenue to manipulate the magnetization of spintronic devices by means of electric signals.

\section{Methods}

$\mathrm{Pt}(3 \mathrm{~nm}) / \mathrm{Co}(0.6 \mathrm{~nm}) / \mathrm{Al}(1.6 \mathrm{~nm})$ and $\mathrm{Pt}(3 \mathrm{~nm}) / \mathrm{Co}(0.6 \mathrm{~nm}) / \mathrm{Pt}(3 \mathrm{~nm})$ layers were deposited on a thermally oxidized silicon wafer by conventional d.c. magnetron sputtering with a base pressure of $10^{-8} \mathrm{mbar}$. Subsequently the $\mathrm{Pt} / \mathrm{Co} / \mathrm{Al}$ samples were oxidized for $35 \mathrm{~s}$ using an oxygen radiofrequency plasma with partial oxygen pressure of $3 \times 10^{-3}$ mbar and $10 \mathrm{~W}$ radiofrequency power. The relatively thick $\mathrm{Al}$ layer ensures the chemical stability of the layers and preserves constant magnetic properties over time, so that no further capping is required to protect the samples. The films so obtained were patterned into an array of wires using electron-beam lithography and Ar ion etching. The width of the wires was fixed at $500 \mathrm{~nm}$ to allow them to be easily imaged using magneto-optical techniques. The length of the wires was chosen to be $5 \mu \mathrm{m}$ so that the device resistance $(\sim 100 \Omega)$ facilitates the injection of current pulses of sufficient intensity. For the same purpose, low-resistance contact pads were fabricated in a second optical lithography step followed by the deposition of a Au layer and lift-off. Magnetic images were obtained using a wide-field magneto-optical Kerr microscope. The external magnetic field was applied using an electromagnet included in the set-up with the field value controlled by a bipolar power supply. The maximum applied field value was $\pm 95 \mathrm{mT}$ corresponding to the voltage range limit of the power supply. The current pulses were generated by a $100 \mathrm{~V} / 2 \mathrm{~A}$ pulse generator. The rise and fall times of the pulses were approximately $6 \mathrm{~ns}$ as measured by an oscilloscope connected in series with the sample. The length of the current pulses was chosen to be $100 \mathrm{~ns}$, almost ten times longer compared with the cumulated rise and fall times, but still short enough to prevent sample damage by overheating or electro-migration. This pulse length is much longer than the characteristic timescale for magnetization dynamics and therefore its action is, from this point of view, similar to that of a continuous current.

Received 3 September 2009; accepted 4 December 2009; published online 10 January 2010

\section{References}

1. Ohno, H. et al. Electric-field control of ferromagnetism. Nature 408, 944-946 (2000).

2. Weisheit, M. et al. Electric field-induced modification of magnetism in thin-film ferromagnets. Science 315, 349-351 (2007).

3. Chu, Y.-H. et al. Electric-field control of local ferromagnetism using a magnetoelectric multiferroic. Nature Mater. 7, 478-482 (2008).

4. Myers, E. B., Ralph, D. C., Katine, J. A., Louie, R. N. \& Buhrman, R. A. Current-induced switching of domains in magnetic multilayer devices. Science 285, 867-870 (1999).

5. Wegrowe, J.-E., Kelly, D., Jaccard, Y., Guittienne, Ph. \& Ansermet, J.-Ph. Current-induced magnetization reversal in magnetic nanowires. Europhys. Lett. 45, 626-632 (1999).

6. Ralph, D. C. \& Stiles, M. D. Spin transfer torques. J. Magn. Magn. Mater. 320, 1190-1216 (2008).

7. Chappert, C., Fert, A. \& Nguyen Van Dau, F. The emergence of spin electronics in data storage. Nature Mater. 6, 813-823 (2007).

8. Katine, J. A. \& Fullerton, E. E. Device implications of spin-transfer torques. J. Magn. Magn. Mater. 320, 1217-1226 (2008).

9. Manchon, A. \& Zhang, S. Theory of nonequilibrium intrinsic spin torque in a single nanomagnet. Phys. Rev. B 78, 212405 (2008).

10. Manchon, A. \& Zhang, S. Theory of spin torque due to spin-orbit coupling. Phys. Rev. B 79, 094422 (2009).

11. Chernyshov, A. et al. Evidence for reversible control of magnetization in a ferromagnetic material by means of spin-orbit magnetic field. Nature Phys. 5, 656-659 (2009).

12. Slonczewski, J. C. Current-driven excitation of magnetic multilayers. J. Magn. Magn. Mater. 159, L1-L7 (1996).

13. Berger, L. Emission of spin waves by a magnetic multilayer traversed by a current. Phys. Rev. B 54, 9353-9358 (1996).

14. Dresselhaus, G. Spin-orbit coupling effects in zinc blende structures. Phys. Rev. 100, 580-586 (1955).

15. Bychkov, Yu. A. \& Rashba, E. I. Properties of a 2D electron gas with lifted spectral degeneracy. J. Exp. Theor. Phys. Lett. 39, 78-81 (1984). 
16. Edelstein, V. M. Spin polarization of conduction electrons induced by electric current in two-dimensional asymmetric electron systems. Solid State Commun. 73, 233-235 (1990).

17. Inoue, J., Bauer, G. E. W. \& Molenkamp, L. W. Diffuse transport and spin accumulation in a Rashba two-dimensional electron gas. Phys. Rev. B 67, $033104(2003)$

18. Silov, A. Yu. et al. Current-induced spin polarization at a single heterojunction. Appl. Phys. Lett. 85, 5929-5931 (2004).

19. Kato, Y. K., Myers, R. C., Gossard, A. C. \& Awschalom, D. D. Current-induced spin polarization in strained semiconductors. Phys. Rev. Lett. 93, 176601 (2004).

20. Awschalom, D. \& Samarth, N. Spintronics without magnetism. Physics 2, 50 (2009).

21. Adagideli, I., Bauer, G. E. W. \& Halperin, B. I. Detection of current-induced spins by ferromagnetic contacts. Phys. Rev. Lett. 97, 256601 (2006).

22. Obata, K. \& Tatara, G. Current-induced domain wall motion in Rashba spin-orbit system. Phys Rev. B 77, 214429 (2008).

23. Cardona, M., Christensen, N. E. \& Fasol, G. Relativistic band structure and spin-orbit splitting of zinc-blende-type semiconductors. Phys. Rev. B 38, 1806-1827 (1988).

24. LaShell, S., McDougall, B. A. \& Jensen, E. Spin splitting of an Au(111) surface state band observed with angle resolved photoelectron spectroscopy. Phys. Rev. Lett. 77, 3419-3422 (1996).

25. Henk, J., Hoesch, M., Osterwalder, J., Ernst, A. \& Bruno, P. Spin-orbit coupling in the L-gap surface states of $\mathrm{Au}(111)$ : Spin-resolved photoemission experiments and first-principles calculations. J. Phys.: Condens. Matter 16, 7581-7597 (2004)

26. Krupin, O. et al. Rashba effect at magnetic metal surfaces. Phys. Rev. B 71, 201403(R) (2005).
27. Rodmacq, B., Manchon, A., Ducruet, C., Auffret, S. \& Dieny, B. Influence of thermal annealing on the perpendicular magnetic anisotropy of $\mathrm{Pt} / \mathrm{Co} / \mathrm{AlO}_{x}$ trilayers. Phys. Rev. B 79, 024423 (2009).

28. Gambardella, P. et al. Giant magnetic anisotropy of single cobalt atoms and nanoparticles. Science 300, 1130-1133 (2003).

29. Ast, C. R. et al. Giant spin splitting through surface alloying. Phys. Rev. Lett. 98, 186807 (2007).

30. Miron, I. M. et al. Domain wall spin torquemeter. Phys. Rev. Lett. 102, 137202 (2009).

\section{Acknowledgements}

We thank A. Bachtold and S. O. Valenzuela for critically reading the manuscript and useful discussions. This work was supported by the European Research Council (Starting Grant 203239). Samples were patterned at the NANOFAB facility of the Institut Néel (CNRS).

\section{Author contributions}

I.M.M., G.G., A.S. and P.G. designed the experiment; I.M.M., G.G., S.A. and B.R. fabricated the samples. I.M.M. carried out the experiments with help from S.P. and J.V.; I.M.M. and P.G. analysed the data and wrote the manuscript. All authors discussed the results and commented on the manuscript.

\section{Additional information}

The authors declare no competing financial interests. Supplementary information accompanies this paper on www.nature.com/naturematerials. Reprints and permissions information is available online at http://npg.nature.com/reprintsandpermissions. Correspondence and requests for materials should be addressed to I.M.M. 


\title{
Current-Driven Spin Torque Induced by the Rashba Effect in a Ferromagnetic Metal Layer
}

\author{
Ioan Mihai Miron ${ }^{1}$, Gilles Gaudin ${ }^{2}$, Stéphane Auffret ${ }^{2}$, Bernard Rodmacq ${ }^{2}$, \\ Alain Schuhl ${ }^{2}$, Stefania Pizzini ${ }^{3}$, Jan Vogel ${ }^{3}$ and Pietro Gambardella ${ }^{1,4}$ \\ ${ }^{1}$ Centre d'Investigació en Nanociència i Nanotecnologia (CIN2-ICN), \\ UAB Campus, E-08193 Barcelona, Spain. \\ ${ }^{2}$ SPINTEC, UMR-8191, CEA/CNRS/UJF/GINP, INAC, F-38054 Grenoble, France. \\ ${ }^{3}$ Institut Néel, CNRS/UJF, B.P. 166, F-38042 Grenoble, France. \\ ${ }^{4}$ Institució Catalana de Recerca i Estudis Avançats (ICREA), E-08100 Barcelona, Spain.
}

\section{Magnetization of $\mathrm{Pt} / \mathrm{Co} / \mathrm{AlO}_{\mathrm{x}}$ and $\mathrm{Pt} / \mathrm{Co} / \mathrm{Pt}$}

The absolute magnetization of $\operatorname{Pt}(3 \mathrm{~nm}) / \mathrm{Co}(0.6 \mathrm{~nm}) / \mathrm{AlO}_{\mathrm{x}}(1.6 \mathrm{~nm})$, hereafter indicated as $\mathrm{Pt} / \mathrm{Co} / \mathrm{AlO}_{\mathrm{x}}$, and $\mathrm{Pt}(3 \mathrm{~nm}) / \mathrm{Co}(0.6 \mathrm{~nm}) / \mathrm{Pt}(3 \mathrm{~nm}), \mathrm{Pt} / \mathrm{Co} / \mathrm{Pt}$, was measured at room temperature by vibrating sample magnetometry, yielding saturation values of $M=1090$ and $1110 \mathrm{kA} / \mathrm{m}$ for $\mathrm{Pt} / \mathrm{Co} / \mathrm{AlO}_{\mathrm{x}}$ and $\mathrm{Pt} / \mathrm{Co} / \mathrm{Pt}$, respectively, close to bulk Co. $M_{z}$ hysteresis loops were probed by the extraordinary Hall effect (EHE), as shown in Fig. 1d, applying an external magnetic field perpendicular to the sample plane. The loops are squared, indicating $100 \%$ out-of-plane remanence. The anisotropy field $\mu_{0} \mathrm{H}_{\mathrm{K}}$, defined as the field required to achieve $90 \%$ magnetic polarization along the hard axis, represents a direct measurement of the

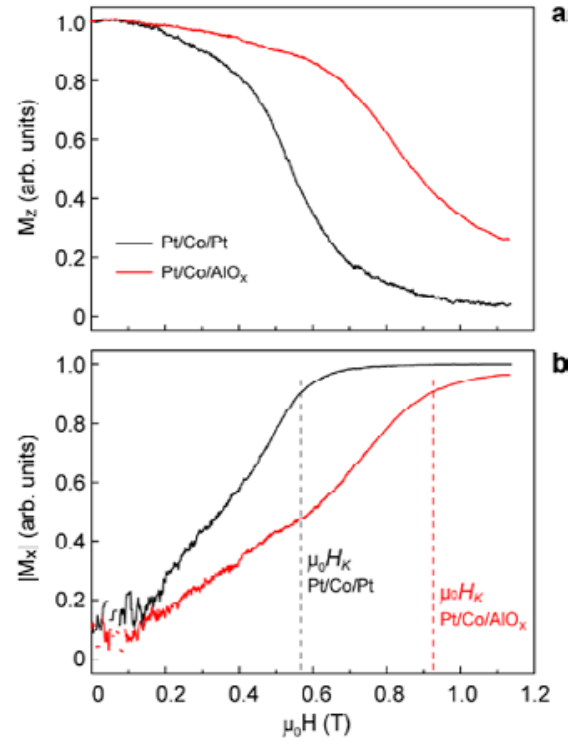

Figure S1: a, EHE measurements of $M_{z}$ as a function of in-plane external field. The EHE values have been normalized to 1 at remanence. $\mathbf{b}$, Inplane $\left|M_{x}\right|$ component calculated from a. The dashed lines indicate the anisotropy field values for $\mathrm{Pt} / \mathrm{Co} / \mathrm{AlO}_{\mathrm{x}}$ (red) and $\mathrm{Pt} / \mathrm{Co} / \mathrm{Pt}$ (black), as defined in the text. 
perpendicular magnetic anisotropy in our system. Following Ref. $1, \mathrm{H}_{\mathrm{K}}$ is obtained by EHE, measuring the decrease of $M_{z}$ due to an in-plane external field (Fig. S1a) and calculating the in-plane magnetization as $\left|M_{x}\right|=\sqrt{1-M_{z}^{2}}$ (Fig. S1b). We find that $\mu_{0} \mathrm{H}_{\mathrm{K}}=0.92 \mathrm{~T}$ in $\mathrm{Pt} / \mathrm{Co} / \mathrm{AlO}_{\mathrm{x}}$ and $0.57 \mathrm{~T}$ in $\mathrm{Pt} / \mathrm{Co} / \mathrm{Pt}$, reflecting the strong magnetocrystalline anisotropy in both layers as well as differences due to the dissimilar $\mathrm{AlO}_{\mathrm{x}}$ and $\mathrm{Pt}$ interfaces.

\section{Kerr microscopy measurements}

To evidence the effects of the Rashba effective field on the magnetic state of the wires the following measurement scheme was devised. An out-of-plane magnetic field was applied prior to each current pulse to saturate the magnetization either upwards or downwards. Due to the high perpendicular anisotropy of both $\mathrm{Pt} / \mathrm{Co} / \mathrm{AlO}_{\mathrm{x}}$ and $\mathrm{Pt} / \mathrm{Co} / \mathrm{Pt}$, the wires magnetization remains fully saturated after removal of the out-of-plane field. The pulse amplitude was first gradually increased to find the domain nucleation threshold at zero field (Fig. S2) by recording Kerr images before and after each pulse. Subsequently, a Kerr reference image was taken and a constant in-plane magnetic field $\mathrm{H}_{\mathrm{ext}}$ applied for 10 seconds. A single $100 \mathrm{~ns}$ current pulse was triggered $5 \mathrm{~s}$ after $\mathrm{H}_{\mathrm{ext}}$ was set. During this period, the magnetic state of the wires was continuously monitored with the Kerr microscope. After removal of $\mathrm{H}_{\mathrm{ext}}$ a second Kerr image was recorded and the differential image obtained as the difference between the second and first image. Note that, because the application of external magnetic fields induces drift of the sample holder and creates disturbing topological contrast in the differential image,

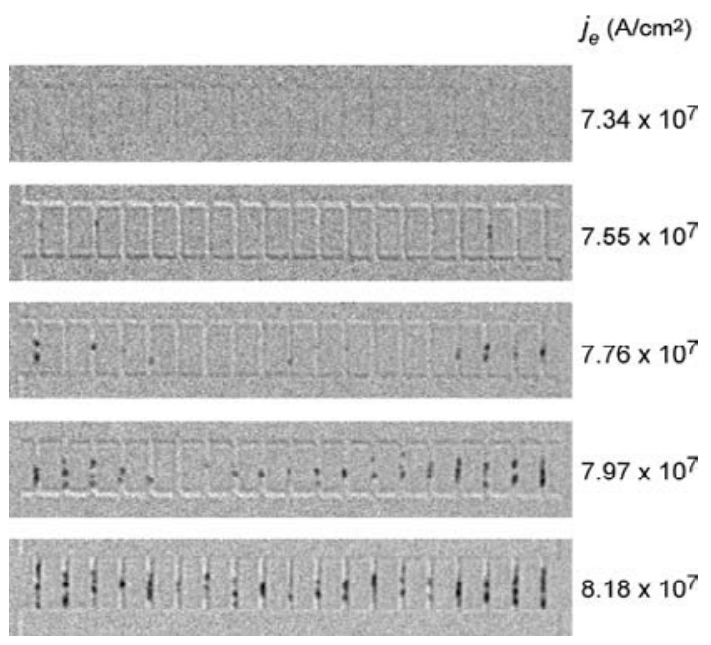

Figure S2: Differential Kerr microscopy images illustrating the gradual onset of domain nucleation in $\mathrm{Pt} / \mathrm{Co} / \mathrm{AlO}_{\mathrm{x}}$ wires induced by current pulses of increasing amplitude at zero field. 
only zero field images were recorded. To ensure that magnetization changes were produced only due to current injection, $\mathrm{H}_{\mathrm{ext}}$ was also applied without triggering the current pulse. The fact that no changes appeared in the magnetization, evidences the essential role played by the current. This is in very good agreement with the high efficiency of the measured Rashbainduced field. For the range of applied currents and magnetic fields used in this experiment, the effective field $\mathrm{H}_{\mathrm{sd}}$ is up to 8 times larger than $\mathrm{H}_{\text {ext. }}$. Therefore the energy barrier for domain nucleation is much lower when $\mathrm{H}_{\mathrm{sd}}$ is present rather than when only $\mathrm{H}_{\mathrm{ext}}$ is applied.

Measurements of the domain nucleation rate carried out on both $\mathrm{Pt} / \mathrm{Co} / \mathrm{AlO}_{\mathrm{x}}$ and $\mathrm{Pt} / \mathrm{Co} / \mathrm{Pt}$ showed reproducible results on different samples.

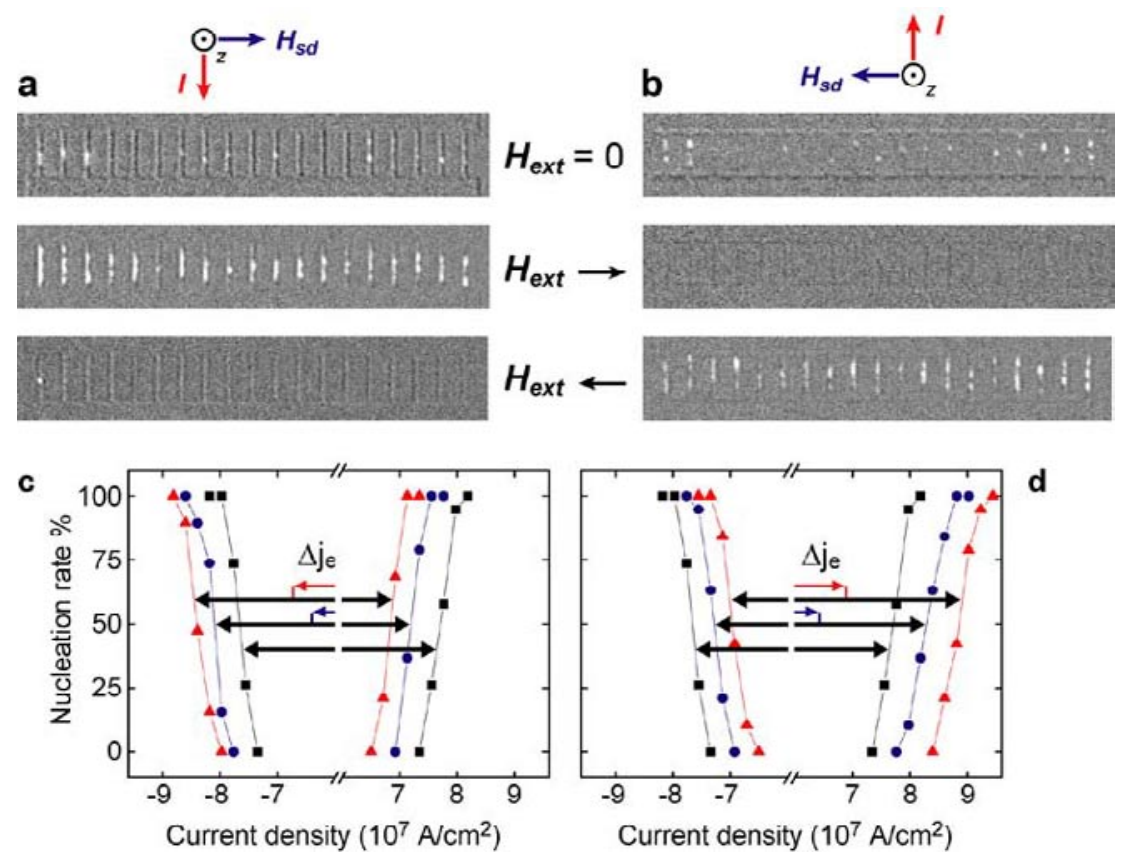

Figure S3: Differential Kerr microscopy images recorded for a, positive and $\mathbf{b}$, negative current pulses in an external field $\mu_{0} \mathrm{H}_{\text {ext }}=0, \pm 47.5 \mathrm{mT}$ applied parallel to $\hat{\mathbf{y}}$ for $\mathrm{Pt} / \mathrm{Co} / \mathrm{AlO}_{\mathrm{x}}$ initially magnetized along the positive $\hat{\mathbf{z}}$ direction. The pulse amplitude corresponds to $7.810^{7} \mathrm{~A} / \mathrm{cm}^{2}$ as in Fig. 1. c, $\mathbf{d}$, Nucleation rate for $\mathbf{H}_{\text {ext }} / / \hat{\mathbf{y}}$ (c) and $\mathbf{H}_{\text {ext }} / /-$ $\hat{\mathbf{y}}(\mathbf{d})$. 


\section{Spin-orbit and spin transfer torques}

It is of interest to analyze the distinctive features of the Rashba torque and effective field $\mathbf{H}_{\text {sd }}$ with respect to other known types of current-induced torques in magnetic materials. The general form of the modified Landau-Lifshitz-Gilbert equation that takes into account this interaction in a single magnetic layer with structure inversion asymmetry (SIA) reads

$$
\frac{\partial \mathbf{M}}{\partial t}=\gamma_{0}\left[\left(\mathbf{H}_{\mathrm{eff}}+\mathbf{H}_{\mathrm{sd}}\right) \times \mathbf{M}\right]+\frac{\alpha}{M_{S}}\left(\mathbf{M} \times \frac{\partial \mathbf{M}}{\partial t}\right)-\frac{1}{\left(1+\beta^{2}\right) M_{S}}(\mathbf{u} \cdot \nabla) \mathbf{M}+\frac{\beta}{\left(1+\beta^{2}\right) M_{S}^{2}} \mathbf{M} \times[(\mathbf{u} \cdot \nabla) \mathbf{M}],
$$

where $\mathbf{M}$ is the local magnetization, $\gamma_{0}$ the gyromagnetic ratio, $\mathbf{H}_{\mathbf{e f f}}$ the magnetic field including contributions from the external, anisotropic, and magnetostatic fields, $\alpha$ the Gilbert damping paramater, and $\beta=\tau_{e x} / \tau_{s f}$ the ratio of the nonadiabatic to adiabatic spin torque, equal to the ratio of the exchange interaction and spin-flip relaxation times ${ }^{2,3}$. Using the same parameter definitions as in the main text, one has

$$
\mathbf{u}=\frac{g \mu_{B} P}{2 e M_{s}} \mathbf{j}_{e} \quad \text { and } \quad \mathbf{H}_{s d}=2 \frac{\alpha_{R} m}{\hbar e M_{S}} P\left(\hat{\mathbf{z}} \times \mathbf{j}_{e}\right) .
$$

From Eq. (1) it follows that the action of $\mathbf{H}_{\mathbf{s d}}$ is analogous to that of an externally applied field, the sign of which depends on the current direction but is independent on the local magnetization orientation.

As it is known from domain wall measurements ${ }^{4}$, the injection of a current into a textured magnetic film produces two additional torque components, called the adiabatic and nonadiabatic spin torques ${ }^{2,3}$, corresponding, respectively, to the third and fourth terms in Eq. (1). Although these two torques exist independently of SIA, recent experiments revealed an enhancement of the nonadiabatic spin torque component in $\mathrm{Pt} / \mathrm{Co} / \mathrm{AlO}_{\mathrm{x}}$ domain walls ${ }^{5}$, which was attributed to the presence of SIA and related increase of the conduction electron spin-flip rate.

There are important differences between the Rashba field $\mathbf{H}_{\mathbf{s d}}$ and the nonadiabatic spin torque enhancement due to SIA. In a single-electron picture, the effect of the Rashba Hamiltonian is to induce the rotation of the spin around an axis perpendicular to the electric field and the electron wavevector. In a many-electron picture, however, the rotation of the spins will have a collective component, equivalent to the Rashba effective field, as well as a dispersive component, owing to the distribution of electron wavevectors on the Fermi sphere. This second component leads to spin decoherence, i.e., a decrease of the spin relaxation time $\tau_{s f}$ Equation (1) shows that the non-adiabatic spin-torque term may be enhanced by the latter 
a) Rashba torque

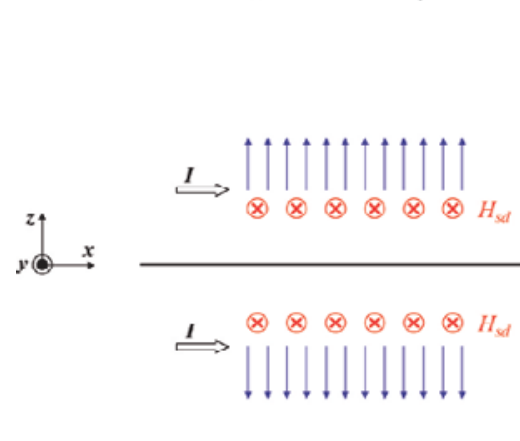

b) Adiabatic and nonadiabatic spin torque in DWs c) Spin torque between noncollinear magnetic layers

Figure S4: Cross-sectional schematic representation of the different types of effective magnetic fields generated by $\mathbf{a}$, Rashba torque, $\mathbf{b}$, adiabatic and nonadiabatic spin torque, and c, spin transfer torque. The bottom panels show the field/torque directions (red) under reversal of the magnetization (blue).

effect, conditional to the presence of a magnetization gradient. Thus, despite their common origin, these two mechanisms are qualitatively and quantitatively different, producing distinct effects on the local magnetization.

As shown in Fig. S4, $\mathbf{H}_{\mathbf{s d}}$ is orthogonal to both current and interface normal, i.e., is a hard axis field parallel to $\hat{\mathbf{y}}$. $\mathbf{H}_{\text {sd }}$ changes sign by reversing the current, is independent of the local magnetization direction (blue arrows), and is homogeneously distributed inside a magnetic film. The field-equivalent of the nonadiabatic spin torque $\left(\mathbf{H}_{\mathrm{NA}}\right)$, on the other hand, acts as an easy axis field directed perpendicular to the layer. Unlike the Rashba field, $\mathbf{H}_{\mathrm{NA}}$ changes sign when the magnetic configuration is reversed and exists only inside a domain wall, vanishing in the case of uniform magnetization. It may be noted as well that the two fields and corresponding torques have opposite time reversal symmetries, with $\mathbf{H}_{\mathbf{s d}}\left(\boldsymbol{\Gamma}_{\mathbf{s d}}\right)$ being odd (even) and $\mathbf{H}_{\mathbf{N A}}\left(\boldsymbol{\Gamma}_{\mathbf{N A}}\right)$ being even (odd) under reversal of both current and magnetization. The difference between these two types of torques reflects also on their magnitude and therefore on their efficacy: for the same current, $\mathbf{H}_{\mathbf{s d}}$ is more then ten times greater compared to the field equivalent of the nonadiabatic spin torque component measured in $\mathrm{Pt} / \mathrm{Co} / \mathrm{AlO}_{\mathrm{x}}(1$ Oe per $\left.1.25 \times 10^{5} \mathrm{~A} / \mathrm{cm}^{2}\right)^{5,6}$.

Finally, we show in Fig. S4c the well-known form of Slonczewski and Berger ${ }^{7,8}$ spin transfer torque occurring when a spin-polarized current traverses two distinct magnetic layers separated by a non-magnetic spacer. In this case, the spin-torque $\left(\Gamma_{\mathrm{ST}}\right)$ does not have a static field analogue, competing with the Gilbert damping in Eq. (1). Similar to the case of domain walls, it only exists if the magnetization directions of the two layers are non-collinear. 
Comparing the different spin torque terms, it is evident that the presence of the Rashba effective field $\mathbf{H}_{\mathbf{s d}}$ offers entirely new possibilities to control the magnetic behavior of thin films and patterned media using an electric current, which are fully complementary and may be integrated with other forms of current-induced torques.

\section{References}

1 Rodmacq, B. Manchon, A. Ducruet, C. Auffret, S. \& Dieny, B. Influence of thermal annealing on the perpendicular magnetic anisotropy of $\mathrm{Pt} / \mathrm{Co} / \mathrm{AlO}_{\mathrm{x}}$ trilayers. Phys. Rev. B 79, 024423 (2009).

2 Zhang, S. \& Li, Z. Roles of nonequilibrium conduction electrons on the magnetization dynamics of ferromagnets, Phys. Rev. Lett. 93, 127204 (2004).

${ }^{3}$ Thiaville, A., Nakatani, Y., Miltat, J. \& Suzuki, Y. Micromagnetic understanding of currentdriven domain wall motion in patterned nanowires. Europhys. Lett. 69, 990-996 (2005).

${ }^{4}$ Beach, G.S.D., Tsoi, M., Erskine, J.L. Current-induc ed domain wall motion J. Magn. Magn. Mater. 320, 1272-1281 (2008).

${ }^{5}$ Miron, I.M. Zermatten, P.-J. Gaudin, G. Auffret, S. Rodmacq, B. \& Schuhl, A. Domain wall spin torquemeter. Phys. Rev. Lett. 102, 137202 (2009).

${ }^{6}$ Note that in Ref. 5 above the presence of the current-induced Rashba field was not contemplated. Considering a posteriori its influence on the determination of the nonadiabatic spin torque on domain walls, it shall be pointed out that an in-plane effective field cannot directly influence Bloch domain wall displacements. However, the Rashba field can in principle have a strong influence on the micromagnetic structure of the domain wall. In such a case, the domain wall width would contract or expand as a function of current by an amount proportional to the square root of the in-plane Rashba field. For the relatively small current densities employed in Ref. $5\left(0.5\right.$ to $\left.2.4 \times 10^{7} \mathrm{~A} / \mathrm{cm}^{2}\right)$, and considering this effect as a correction to the magnetic anisotropy, the estimated domain wall width would change by a maximum of $12 \%$, leading to an analogous variation of the reported nonadiabatic spin-torque efficiency (beta parameter).

7 Slonczewski, J. C. Current-driven excitation of magnetic multilayers. J. Magn. Magn. Mater. 159, L1-L7 (1996).

${ }^{8}$ Berger, L. Emission of spin waves by a magnetic multilayer traversed by a current. Phys. Rev. B 54, 9353-9358 (1996). 